\title{
The Diabetes-Bone Relationship
}

\section{Laura R. McCabe*}

Departments of Physiology and Radiology, Michigan State University, USA

Diabetes is a chronic metabolic disease characterized by hyperglycemia due to the inability of insulin dependent cells to absorb glucose effectively. Either insulin deficiency due to the loss of pancreatic beta cells (type 1 diabetes) or insulin insensitivity/resistance (type 2 diabetes) results in diabetes. According to the World Health Organization, more than 340 million people worldwide have diabetes. Because of the significant metabolic changes that occur in diabetes it is not surprising that most organs in the body become affected to some extent. This in part due to the extended lifespan of patients and the difficulty of maintaining absolute metabolic control even with all the current medical advances. While neuropathy, nephropathy, retinopathy and cardiovascular disease are commonly thought of as consequences of diabetes, it is also known that diabetes can impact the skeleton and cause bone loss and/or altered bone matrix and strength [1]. These changes can also impact bone marrow cells, which are involved in immunity, inflammation and tissue healing. Work over the past several years further supports that bone can also contribute to the regulation of insulin secretion and metabolism [2]. Thus, understanding the mechanisms accounting for diabetic bone pathology is important for developing therapies that could restore bone as well as metabolic and overall patient health and quality of life.

Both type 1 and type 2 diabetes are known to have significant effects on bone health [3]. Type 1 diabetes is associated with bone loss in males and females and at several skeletal sites [4]. In animal models, type 1 diabetes is associated with bone loss, decreased osteoblast activity, increased bone marrow adiposity, osteoblast death and marrow inflammation $[5,6]$. While genetics can influence the amount of bone lost, reports using type 1 diabetic animal models demonstrate that all display bone loss regardless of method of diabetes induction (spontaneous versus pharmacologic [6,7] or strain. However, differences in mouse genetic backgrounds did affects the magnitude of bone loss in response to type 1 diabetes, possibly as a result of differences in bone turnover rates and/or additional metabolic factors. While bone anabolic properties are decreased in patients (similar to animal models), marrow adiposity does not appear to associate with type 1 diabetes in humans but is interestingly linked to serum lipid levels [8]. Type 2 diabetes, on the other hand, is not always associated with bone loss but is associated with reduced bone strength and fracture risk; this is likely due modifications in the bone matrix through hyperglycemia induced glycation.

Both type 1 and type 2 diabetes lead to increased fracture risk [9], making diabetic bone loss a complication that should be considered for monitoring and therapeutic prevention. First line approaches include exercise/loading of bones, vitamin $\mathrm{D}$ and calcium to enhance and/or maintain bone density. Given that most pharmacologic approaches for bone loss reduce catabolic activity by osteoclasts and knowing that diabetes (especially type 1) can suppress catabolic and anabolic properties, diabetic treatment options need to be considered carefully. Ikeda et al. [10] present data showing that, at least in type 2 diabetic patients, the use of alendronate (a bisphosphonate) effectively reduces bone loss. The long-term effects of bisphosphonates on bone density and ultimate strength in type 1 diabetic patients is less well known. An important area of research is testing the efficacy of existing osteoporotic treatments in diabetic patients to assess their impact on bone health in this population. Identifying existing drugs that promote bone loss in patients is also important. For example, anti-diabetic compounds that activate PPAR $\gamma$ (i.e., rosiglitazone) to lower hyperglycemia, hyperinsulinemia and hypertriglyceridemia in type 2 diabetic patients, also induce marrow adiposity, bone loss and increase fracture risk. Several therapeutic possibilities are currently being examined. In this issue, Devesh et al. [11] identified that combination therapy of pioglitazone with spirulina reduced the risk of fracture in insulin resistance rats and that spirulina complemented the anti-hyperglycemic and anti lipidemic activity of pioglitazone. It is important to continue to examine factors (existing or novel) that can serve as therapeutics to benefit diabetic bone health.

As noted previously, it is imperative that the mechanisms accounting for diabetic bone loss be identified to aid in the development of therapeutic targets and novel treatment regimes. It is well known that diabetes leads to decreased insulin signaling and that diabetes can impact IGF-1 signaling as well; both factors are known to have bone anabolic properties and alterations in their signaling pathways can contribute to diabetic bone pathology [12]. In addition, hyperglycemia itself can alter cellular metabolic processes as well as directly modify proteins through advanced glycation end products (AGEs). AGE and reactive oxygen species can impact osteoblast activity and likely contribute to diabetic bone pathology. The underlying mechanism is thought to involve AGE signaling through their receptor RAGE to impact cell function and inflammation. Witzke and Vinik [13] discuss the role of RAGE in osteoclast activation in Charcot neuroarthropathy, which can occur in diabetic patients. Therefore, administration of sRAGE in people who lack adequate endogenous production might have the anti-resportive potential by interrupting the osteoclastic activation.

In summary, both type 1 and type 2 diabetes is associated with increased fracture risk through similar as well as potentially different mechanisms. With medical advances lengthening patient lifespan. This leads to extended time of tissue exposure to diabetic conditions (altered metabolism) and compounds disease complications with those of aging. Understanding the factors/mechanisms involved in the diabetic bone pathologies is critical to optimizing current treatments and identifying new drug treatment targets and therapies.

\section{References}

1. McCabe L, Zhang J, Raehtz S (2011) Understanding the skeletal pathology of type 1 and 2 diabetes mellitus. Crit Rev Eukaryot Gene Expr 21: 187-206.

*Corresponding author: Laura R. McCabe, Departments of Physiology and Radiology, Michigan State University, 2201 Biomedical Physical Science Bldg., East Lansing, MI 48824, USA, Tel: (517) 884-5152; Fax: (517) 355-5125; E-mail: mccabel@msu.edu

Received July 27, 2012; Accepted August 06, 2012; Published August 10, 2012

Citation: McCabe LR (2012) The Diabetes-Bone Relationship. J Diabetes Metab S1:e001. doi:10.4172/2155-6156.S1-e001

Copyright: (c) 2012 McCabe LR. This is an open-access article distributed under the terms of the Creative Commons Attribution License, which permits unrestricted use, distribution, and reproduction in any medium, provided the original author and source are credited. 
2. Motyl KJ, McCabe LR, Schwartz AV (2010) Bone and glucose metabolism: a two-way street. Arch Biochem Biophys 503: 2-10

3. Peter Vestergaard (2011) Diabetes and Bone. J Diabetes Metab S1-001.

4. Hofbauer LC, Brueck CC, Singh SK, Dobnig H (2007) Osteoporosis in Patients with Diabetes Mellitus. J Bone Miner Res 22: 1317-1328.

5. Coe LM, Irwin R, Lippner D, McCabe LR (2011) The bone marrow microenvironment contributes to type I diabetes induced osteoblast death. J Cell Physiol 226: 477-483.

6. McCabe LR (2007) Understanding the pathology and mechanisms of type I diabetic bone loss. J Cell Biochem 102: 1343-1357.

7. Botolin S, McCabe LR (2007) Bone loss and increased bone adiposity in spontaneous and pharmacologically induced diabetic mice. Endocrinology 148 198-205.

8. Slade JM, Coe LM, Meyer RA, McCabe LR (2012) Human bone marrow adiposity is linked with serum lipid levels not T1-diabetes. J Diabetes Complications 26: 1-9.

9. Shivaswamy V, Mack L, Larsen J (2011) Diabetes, Bone Density, and Fractures. J Diabetes Metab S1-004.

10. Ikeda T, Iwata K (2011) Long-Term Effect of Alendronate on Bone Minera Density in Postmenopausal Type 2 Diabetes Mellitus. J Diabetes Metab S1002.

11. Devesh C, Kritika M, Anroop N, Prabodh Kumar S, Sumeet G (2012) Spirulina Reverses Histomorphological Changes in Diabetic Osteoporosis in Pioglitazone Treated Rats. J Diabetes Metab S1-006.

12. Fowlkes JL, Bunn RC, Thrailkill KM (2011) Contributions of the Insulin/InsulinLike Growth Factor-1 Axis to Diabetic Osteopathy. J Diabetes Metab S1-003.

13. Witzke KA, Vinik Al (2012) Charcot Neuroarthropathy may be the RAGE. J Diabetes Metab S1-005.
This article was originally published in a special issue, Diabetic Osteoporosis handled by Editor(s). Dr. Laura McCabe, Michigan State University, USA 